\title{
Determinants of stunting and severe stunting among under-fives in Tanzania: evidence from the 2010 cross-sectional household survey
}

\author{
Lulu Chirande ${ }^{1}$, Deborah Charwe ${ }^{2}$, Hadijah Mbwana ${ }^{3}$, Rose Victor², Sabas Kimboka², Abukari Ibrahim Issaka ${ }^{6}$, \\ Surinder K. Baines ${ }^{4}$, Michael J. Dibley ${ }^{5}$ and Kingsley Emwinyore Agho ${ }^{6^{*}}$
}

\begin{abstract}
Background: Stunting is one of the main public health problems in Tanzania. It is caused mainly by malnutrition among children aged less than 5 years. Identifying the determinants of stunting and severe stunting among such children would help public health planners to reshape and redesign new interventions to reduce this health hazard. This study aimed to identify factors associated with stunting and severe stunting among children aged less than five years in Tanzania.

Methods: The sample is made up of 7324 children aged 0-59 months, from the Tanzania Demographic and Health Surveys 2010. Analysis in this study was restricted to children who lived with the respondent (women aged 15-49 years). Stunting and severe stunting were examined against a set of individual-, household- and community-level factors using simple and multiple logistic regression analyses.
\end{abstract}

Results: The prevalence of stunting and severe stunting were $35.5 \%$ [95 \% Confidence interval (Cl): 33.3-37.7] and 14.4 \% (95 \% Cl: 12.9-16.1) for children aged 0-23 months and $41.6 \%$ (95 \% Cl: 39.8-43.3) and $16.1 \%$ (95 \% Cl: 14.8-17.5) for children aged 0-59 months, respectively. Multivariable analyses showed that the most consistent significant risk factors for stunted and severely-stunted children aged 0-23 and 0-59 months were: mothers with no schooling, male children, babies perceived to be of small or average size at birth by their mothers and unsafe sources of drinking water [adjusted odds ratio (AOR) for stunted children aged 0-23 months $=1.37 ; 95 \% \mathrm{Cl}:(1.07,1.75)$ ]; [AOR for severely stunted children aged 0-23 months $=1.50 ; 95 \% \mathrm{Cl}:(1.05,2.14)]$, [AOR for stunted children aged 0-59 months $=1.42 ; 95 \% \mathrm{Cl}$ : $(1.13,1.79)]$ and [AOR for severely stunted children aged 0-59 months $=1.26 ; 95 \% \mathrm{Cl}$ : $(1.09,1.46)]$.

Conclusions: Community-based interventions are needed to reduce the occurrence of stunting and severe stunting in Tanzania. These interventions should target mothers with low levels of education, male children, small- or average-size babies and households with unsafe drinking water.

Keywords: Stunting, Under-fives, Deaths, Undernutrition, Tanzania

\section{Background}

Stunting arises as a result of chronic restriction of a child's potential growth brought about by the cumulative effects of inadequate food intake and poor health conditions that result from endemic poverty [1]. This restricted growth is an important cause of morbidity and mortality in infants

\footnotetext{
* Correspondence: K.Agho@uws.edu.au

${ }^{6}$ School of Science and Health, Western Sydney University, Building 24.2.40, Campbelltown Campus, Locked Bag 1797, Penrith, NSW 2571, Australia

Full list of author information is available at the end of the article
}

and children $[2,3]$. Poor socioeconomic conditions and an increased risk of frequent and early exposure to adverse conditions, such as illness or inappropriate feeding practices may give rise to high levels of stunting. A decline in the national stunting rate is usually an indication of improvements in the overall socioeconomic conditions of a country [4]. The global variation of the prevalence of stunting is considerable, ranging from 5 to $65 \%$ among the less-developed countries [5]. In developing countries, the prevalence of stunting starts to rise at about three months of age and then slows at around two years of age [5]. 
According to Black et al. [3], more than one-third of child deaths and more than $10 \%$ of the total global disease burden are attributed to maternal and child undernutrition, which may result in stunting among others. The global burden of stunting is enormous, with approximately 195 million occurring in the developing world [5]. Many developing countries report far higher rates of stunting prevalence than any other illnesses due to child undernutrition, making it an important public health issue.

Among the different regions of Africa, the decline in stunting has been found to be greatest in the northern and middle parts. However, the prevalence has hardly changed in the other (eastern, western, and southern) subregions of the continent [6]. It is estimated that there are presently 171 million stunted preschool children worldwide, of which approximately $98 \%$ reside in developing countries and about $35 \%$ in Africa. Due to expanding population, the number of stunted pre-school children in Africa as a whole increased from 51 million in 2000 to 60 million in 2010, and if present trends do not change, these numbers are reported to further increase to 64 million in 2020 [6].

According to the 2010 Tanzania Demographic and Health Survey (TDHS), $42 \%$ of Tanzanian children aged less than five years are stunted [7] and places Tanzania among the 10 worst-affected countries in the world. In spite of a reduction from $48 \%$ (1996) to $42 \%$ (2010), the prevalence of child stunting in Tanzania in 2010 was still 'unacceptably high', by World Health Organization (WHO) standards and greater efforts are thus required to decrease the prevalence of stunting among Tanzanian children.

Factors that may indirectly influence stunting levels among children in developing countries include socioeconomic status such as mother's education and occupation, household income and health expenditure [8-10]. In addition, factors such as micronutrient deficiencies, inadequate protein intake and infections may directly cause stunting $[11,12]$. There have been several studies on risk factors for stunting from different countries. For instance, a study on the magnitude and determinants of stunting in children aged 5 years or younger in food surplus region of Ethiopia found males, children aged less than 7 months and children who contracted diarrhoea to be significantly more likely to be stunted [13].

Another study on the determinants of linear growth and predictors of severe stunting during infancy in rural Malawi found the risk factors for severe stunting to be: preterm birth $(<37$ gestational weeks), maternal short stature $(<160 \mathrm{~cm})$, maternal failure to gain $>200 \mathrm{~g} /$ week during pregnancy, home delivery and paternal illiteracy [14] These studies, however, have been limited in scope as they were not population-based. In Tanzania, there have been few recent studies on factors associated with stunting among children. These studies, however, have been limited in scope. For instance, a recent crosssectional study [15] conducted in Tanzania revealed that low birth weight and low BMI of mothers were the strong predictors of stunting among children. This study covered only one district - the Kilosa district. Another recent Tanzanian cross-sectional study [16] used multivariate logistic regression model to show that maternal education and child's age were independent predictors of stunting. This study also covered just one district - the Same district of the Kilimanjaro region of Tanzania. Thus, there has not been any recent population-based study that has investigated risk factors of stunting in Tanzania. This study therefore aimed to identify and discuss factors associated with stunting and severe stunting among children aged 5 years or younger, using the latest TDHS dataset. Results of this study would contribute to the extant literature and enable policy makers to institute interventions to minimise the burden of stunting and severe stunting in Tanzanian children.

\section{Ethics}

This study was based on an analysis of existing public domain survey datasets that are freely available online with all identifier information removed. The survey was approved by the Ethics Committee of the ICF Macro at Calverton in the USA and by the Ethics Committee in Tanzania. Written consent was obtained from all respondents and all information was collected confidentially.

\section{Methods}

\section{Data sources}

The data examined were from the 2010 Tanzania Demographic and Health Survey (TDHS 2010). The survey involved completed interviews of 10,139 ever-married women aged 15-49 years and utilised three questionnaires: a household, women's and men's questionnaire. The survey collected anthropometric data for all sampled children in Tanzania; including those who were not biological offsprings of the women interviewed in the survey. Each trained interviewer carried a scale and measuring board. The scales were lightweight, bathroom-type with a digital screen. Recumbent heights were measured for children aged less than 24 months whilst the standing heights of older children were measured. The present analysis was restricted to the children aged 0-59 months, living with the respondent and alive. The total weighted sample size was 7324 , and the survey yielded a response rate of $96.4 \%$.

To determine their risk factors, the outcome variables (stunting and severe stunting), were examined against a set of individual-, household- and community-level factors. Individual-level factors included variables from attributes of the parents, infant and mother-infant dyad. Household wealth index and source of drinking water constituted the household-level factors while community- 
level factors were type of residence (urban or rural) and geographical zones.

Household wealth index was calculated as a score of household assets such as ownership of means of transport, ownership of durable goods and household facilities, which was weighted using the principal components analysis method [17]. This index was divided into five categories (quintiles), and each household was assigned to one of these categories. In the TDHS datasets, household wealth index variable was categorized into five quintiles (poorest, poorer, middle, richer and richest).

\section{Statistical analyses}

To determine the level of stunting and severe stunting in children aged 0-23 months and 0-59 months, the dependent variable was expressed as a dichotomous, that is, category 0 (not stunted (>-2SD) or not severely stunted (>-3SD) and category 1 (stunted (>-2SD) or severely stunted (>-3SD).

Analyses were performed using Stata version 12.1 (StataCorp, College Station, TX, USA). 'Svy' commands were used to allow for adjustments for the cluster sampling design, sampling weights and the calculation of standard errors. The Taylor series linearization method was used in the surveys to estimate confidence intervals (CIs) around prevalence estimates. The chi-squared test was used to test the significance of associations. Multiple logistic regression was used to adjust for the complex sampling design and weights. Univariate binary logistic regression analysis was performed to examine the association between stunted and severely stunted children aged 0-23 months and overall stunted children aged 0-59 months.

In the multivariable analysis models, a manual procedure of stepwise backward elimination process was used to identify factors that were significantly associated with the study outcomes using $5 \%$ significance level. In order to avoid or minimise any statistical error in our analysis, we repeated the manual procedure of stepwise backward elimination process by using a different approach. This involved three steps: (1) only potential risk factors with $P$-value $<0.20$ were entered in the backward elimination process, (2) the backward elimination was tested by including all variables (all potential risk factors); and, (3) Any collinearity was tested and reported in the final model. The odds ratios with $95 \%$ CIs were calculated in order to assess the adjusted risk of independent variables, and those with $P<0.05$ were retained in the final model.

\section{Results}

\section{Characteristics of the sample}

Of the total sample of 7234 children aged 0-59 months, the majority lived in rural areas $(80.3 \%)$. Approximately $84 \%$ of the interviewed mothers were employed in the past 12 months, and $6.2 \%$ had secondary education or higher. Of the total births, $49.7 \%$ took place at a health facility. Only a small proportion of deliveries (4.3\%) took place by caesarean section. Male (49.8\%) and female $(50.2 \%)$ children were nearly equally represented in the sample. About $99 \%$ of mothers had made at least one antenatal clinic visit during pregnancy, and $45.2 \%$ of the mothers were aged $25-34$ years. About $12 \%$ of children were exclusively breastfed and $47.8 \%$ of children were breastfed in addition to being given supplements. According to the mothers' perception, $70.6 \%$ of children were of average size, $7.9 \%$ were of small or very small size and $29.5 \%$ were of large size at birth. Nearly $42 \%$ of mothers could not read a sentence. About $21 \%$ of children lived in the Western geographical zone and $20.3 \%, 13.9 \%$ and $2.7 \%$ of children lived in the Lake, Southern Highlands and Zanzibar regions respectively (Table 1 ).

As illustrated in Fig. 1, the prevalence of stunted children aged 0-23 months and 0-59 months was 16 and $42 \%$ respectively. The overall prevalence of severely stunted children aged 0-23 months and 0-59 months was 14 and $35 \%$, respectively.

\section{Multivariate analyses}

Tables 2 and 3 show the unadjusted and adjusted ORs for the association between stunted and severely stunted children and child-, household- and community-level characteristics of children aged 0-23 and children aged 0-59 months.

\section{Risk factors for stunting}

Table 2 shows factors that posed risk to stunting among children aged 0-23 months and those aged 0-59 months. Increased child age was found to be statistically associated with stunted children aged 0-23 months. The risk of stunting was significantly higher among male children compared to females for both age brackets. Children who were perceived by their mothers to be very small or small at birth were significantly more likely to be stunted than those who were perceived to be large. Babies delivered by younger mothers (aged less than 20 years) were significantly more likely to be stunted compared to those delivered by mothers aged 20-29 years. The odds for stunting among children of both age brackets increased significantly among those who lived in households with no access to potable water and for those whose fathers had limited or no schooling and worked in an agricultural industry. Children who were delivered at home, who were delivered by traditional birth attendants (TBAs), whose mothers did not have any antenatal clinic visits and those whose mothers had a Body Mass index (BMI) of less than $18.5 \mathrm{kgm}^{-2}$ were significantly more likely to be stunted. The risk of stunting was also found to be significantly high among children who were given 
Table 1 Characteristics of parents and children aged 0-59 months in Tanzania $2010(n=7324)$

\begin{tabular}{|c|}
\hline Characteristic \\
\hline Individual level factors \\
\hline Parental factor \\
\hline Maternal working status \\
\hline Non-working \\
\hline $\begin{array}{l}\text { Working } \\
\text { (past } 12 \text { months) }\end{array}$ \\
\hline Maternal education \\
\hline No education \\
\hline Primary \\
\hline Secondary and above \\
\hline Partner's occupation \\
\hline Non agriculture \\
\hline Agriculture \\
\hline Not working \\
\hline $\begin{array}{l}\text { Partner's education } \\
(n=6932)\end{array}$ \\
\hline No education \\
\hline Primary \\
\hline Secondary and above \\
\hline Mother's age \\
\hline $15-24$ years \\
\hline $25-34$ years \\
\hline $35-49$ years \\
\hline Mother's age at birth \\
\hline$<19$ years \\
\hline 20-29 years \\
\hline 30-39 years \\
\hline 40 and above \\
\hline
\end{tabular}

Marital status

$$
\begin{aligned}
& \text { Currently married } \\
& \text { Formerly married } \\
& \text { (div/sep/widow) } \\
& \text { Never married }
\end{aligned}
$$

Birth order

$$
\begin{aligned}
& \text { First-born } \\
& \text { 2nd -4th } \\
& 5 \text { or more }
\end{aligned}
$$

Preceding birth interval

$$
\text { No previous birth }
$$

$<24$ months

$>24$ months

Place of delivery

Home

Health facility

1887

456

2168

4759

398

1266

5090

576

2188

3310

1826

1091

3805

2141

288

6260

1439

3546

2339

895

4979

3684

3640

n $\%$

$\%$

Mor

Mode of delivery $(n=7301)$

$\begin{array}{lll}\text { Non-caesarean } & 6987 & 95.7 \\ \text { Caesarean } & 314 & 4.3\end{array}$

Type of delivery assistance

$(n=7193)$

$984 \quad 13.4$

$6340 \quad 86.6$

Health professional

$3567 \quad 49.6$

Traditional birth attendant

$976 \quad 13.6$

Relatives and other untrained personnel

$2388 \quad 33.2$

25.8

No one

262

$4982 \quad 68.0$

Antenatal clinic visits

$(n=5134)$

None

98

1-3.

2839

55.3

$4+$

2198

42.8

5.4

Timing of postnatal check-up $(n=7235)$

No check-ups (including missing)

$5536 \quad 76.5$

18.3

$0-2$ days

$814 \quad 11.2$

73.4

3-6 days

$7+$ days

$327 \quad 4.5$

$559 \quad 7.7$

Maternal BMI $(n=7240)$

29.9

$<=18.5(\mathrm{~kg} / \mathrm{m} 2)$

$668 \quad 9.2$

45.2

$>18.5(\mathrm{~kg} / \mathrm{m} 2)$

6572

90.8

24.9

Child breastfeeding (BF) status

Exclusive BF

$840 \quad 11.5$

14.9

BF + water

177

2.4

52.0

BF + supplements ${ }^{\mathrm{a}}$

3499

47.8

No BF

2809

38.4

3.9

Mother is literate $(n=7257)$

No

3024

41.7

85.5

Yes

4233

58.3

$701 \quad 9.6$

Mother read newspaper $(n=7317)$

No

6418

87.7

Yes

900

19.6

Mother listened to the radio $(n=7322)$

48.4

No

3537

48.3

31.9

Yes

3785

51.7

Mother watched TV

No

6342

86.6

12.2

Yes

982

13.4

68.1

Child level factors

Sex of baby

50.3

Male

3647

49.8

49.7

Female

3678 
Table 1 Characteristics of parents and children aged 0-59 months in Tanzania 2010 ( $n=7324)$ (Continued)

\begin{tabular}{|c|c|c|}
\hline \multicolumn{3}{|l|}{ Size of baby } \\
\hline Small & 562 & 7.9 \\
\hline Average & 4992 & 70.6 \\
\hline Large & 1522 & 21.5 \\
\hline \multicolumn{3}{|c|}{ Child had diarrhoea in the last 2 weeks $(n=7308)$} \\
\hline No & 6207 & 84.9 \\
\hline Yes & 1101 & 15.1 \\
\hline \multicolumn{3}{|c|}{ Child had fever in last two weeks $(n=7303)$} \\
\hline No & 5560 & 76.1 \\
\hline Yes & 1743 & 23.9 \\
\hline \multicolumn{3}{|l|}{ Household level factors } \\
\hline \multicolumn{3}{|l|}{ Wealth Index } \\
\hline Poorest & 1566 & 21.4 \\
\hline Poorer & 1747 & 23.9 \\
\hline Middle & 1647 & 22.5 \\
\hline Rich & 1369 & 18.7 \\
\hline Richest & 996 & 13.6 \\
\hline \multicolumn{3}{|l|}{ Source of drinking water } \\
\hline Protected & 3088 & 42.2 \\
\hline Unprotected & 4237 & 57.8 \\
\hline \multicolumn{3}{|l|}{ Community level factors } \\
\hline \multicolumn{3}{|l|}{ Type of residence } \\
\hline Urban & 1442 & 19.7 \\
\hline Rural & 5883 & 80.3 \\
\hline \multicolumn{3}{|l|}{ Geographic Zones } \\
\hline Northern & 956 & 13.1 \\
\hline Eastern & 849 & 11.6 \\
\hline Western & 1547 & 21.1 \\
\hline Southern Highlands & 1016 & 13.9 \\
\hline Lake & 1487 & 20.3 \\
\hline Southern & 567 & 7.7 \\
\hline Central & 708 & 9.7 \\
\hline Zanzibar & 196 & 2.7 \\
\hline
\end{tabular}

${ }^{\mathrm{a}} \mathrm{BF}+$ supplements included $\mathrm{BF}+$ liquids/juice; $\mathrm{BF}+$ other milk and $\mathrm{BF}+$ complementary foods

supplements in addition to breast milk and as well as those who were non-breastfed. Other risk factors associated with stunting were rural children, children from the poorest households, children whose mothers were illiterate, in paid employment and resided in the Southern Highlands zone of Tanzania.

\section{Risk factors for severe stunting}

Table 3 shows the risk factors associated with severe stunting among children aged 0-59 months. Male children and babies perceived by their mothers to be small

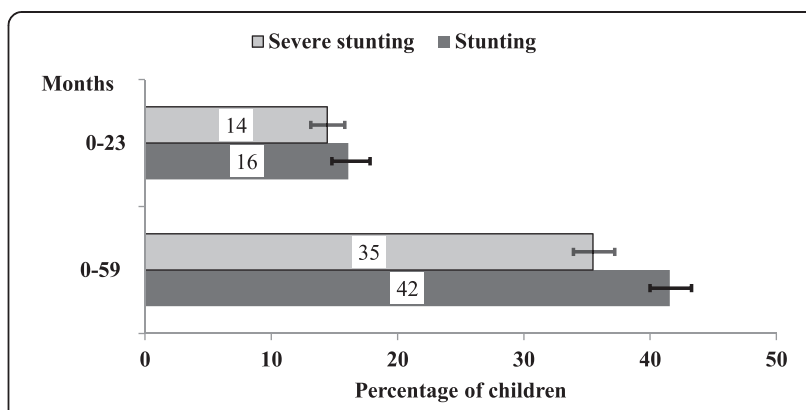

Fig. 1 Prevalence of stunting and severe stunting in children aged $0-23$ and $0-59$ months

at birth were significantly more likely to be severely stunted compared to females and babies perceived to be of medium or large size at birth. The risk of severe stunting was significantly higher among children whose parents had no schooling and were illiterate. Children from poorest households, those who resided in urban areas and in the Northern zone of Tanzania were significantly more likely to become severely stunted. The risk of severe stunting was significantly higher among children who were delivered at home by Traditional Birth Attendants (TBAs) and whose mothers did not attend any antenatal clinics. Children who were $5^{\text {th }}$-born or higher, children who were perceived by their mothers to be small at birth and those from poorest households with no potable drinking water were significantly associated with severe stunting (Table 3).

\section{Discussion}

The present paper was designed to determine factors associated with stunting and severe stunting among Tanzania children aged 0-59 months. The main risk factors for stunting in the study were: age of the child, child's sex, maternal level of educational, perceived size of the child at birth, mother's age at child's birth, place of delivery, type of birth delivery assistance, maternal BMI and breastfeeding status of a child. Factors associated with severe stunting included: sex of the child, parent's level of education and literacy, household wealth index, place of delivery and type of delivery assistance. Birth order of the child, perceived size of the baby at birth, source of drinking water and geographical region were also factors significantly associated with severe stunting.

The main strengths of our study were that it used a nationally-representative survey data and applied appropriate statistical adjustments for the cluster sampling design in the analysis. Our analysis was able to determine the most vulnerable age group and the modifiable characteristics that affected stunting in a large sample size. One key limitation, however, was that we could not establish the cause and effect relationships; because of the cross-sectional nature of the study design. In addition, 
Table 2 Factors associated with stunting in children aged 0-23 months and 0-59 months

\begin{tabular}{|c|c|c|c|c|c|c|c|c|}
\hline \multirow[t]{2}{*}{ Characteristic } & \multicolumn{4}{|c|}{ Stunted children 0-23 Months } & \multicolumn{4}{|c|}{ Stunted children 0-59 Months } \\
\hline & $\begin{array}{l}\text { Unadjusted OR } \\
{[95 \% \mathrm{Cl}]}\end{array}$ & $P$ & $\begin{array}{l}\text { Adjusted OR } \\
{[95 \% \mathrm{Cl}]}\end{array}$ & $P$ & $\begin{array}{l}\text { Unadjusted OR } \\
{[95 \% \mathrm{Cl}]}\end{array}$ & $p$ & $\begin{array}{l}\text { Adjusted OR } \\
{[95 \% \mathrm{Cl}]}\end{array}$ & $p$ \\
\hline \multicolumn{9}{|l|}{ Parental factor } \\
\hline \multicolumn{9}{|l|}{ Maternal working status } \\
\hline Non-working & 1.00 & & & & 1.00 & & & \\
\hline $\begin{array}{l}\text { Working } \\
\text { (past } 12 \text { months) }\end{array}$ & 1.57 [1.19-2.07] & 0.002 & & & $1.23[1.02-1.49]$ & 0.029 & & \\
\hline \multicolumn{9}{|l|}{ Maternal education } \\
\hline Secondary and above & 1.00 & & 1.00 & & 1.00 & & 1.00 & \\
\hline Primary & $2.08[1.33-3.26]$ & 0.001 & $1.82[1.15-2.86]$ & 0.011 & $2.26[1.61-3.18]$ & $<0.0001$ & $1.53[1.07-2 . .19]$ & 0.019 \\
\hline No education & $2.51[1.59-3.96]$ & $<0.001$ & $2.26[1.41-3.60]$ & 0.001 & $2.54[1.77-3.64]$ & $<0.0001$ & $1.61[1.08-2.40]$ & 0.019 \\
\hline \multicolumn{9}{|l|}{ Partner's occupation } \\
\hline Not working & 1.00 & & 1.00 & & 1.00 & & & \\
\hline Agriculture & $1.57[1.06-2.30]$ & 0.023 & $1.62[1.05-2.49]$ & 0.027 & $1.42[1.21-1.67]$ & $<0.001$ & & \\
\hline Non agriculture & $1.07[0.72-1.59]$ & 0.712 & $1.30[0.80-2.04]$ & 0.233 & $0.98[0.72-1.32]$ & 0.889 & & \\
\hline \multicolumn{9}{|l|}{ Partner's education } \\
\hline Secondary and above & 1.00 & & & & 1.00 & & & \\
\hline Primary & $2.19[1.44-3.03]$ & $<0.001$ & & & $2.08[1.58-2.14]$ & $<0.001$ & & \\
\hline No education & $1.74[1.12-2.72]$ & 0.014 & & & $2.02[1.51-2.71]$ & $<0.001$ & & \\
\hline \multicolumn{9}{|l|}{$\begin{array}{l}\text { Mother's age } \\
\text { (years) }\end{array}$} \\
\hline $15-24$ & 1.00 & & & & 1.00 & & & \\
\hline $25-34$ & $0 . .86[0.70-1.05]$ & 0.145 & & & $0.92[0.80-1.06]$ & 0.246 & & \\
\hline $35-49$ & $1.20[0.93-1.55]$ & 0.168 & & & $1.10[0.93-1.31]$ & 0.277 & & \\
\hline \multicolumn{9}{|c|}{$\begin{array}{l}\text { Mother's age at child's birth } \\
\text { (years) }\end{array}$} \\
\hline $20-29$ & 1.00 & & 1.00 & & 1.00 & & & \\
\hline $30-39$ & $1.21[0.95-1.53]$ & 0.111 & $1.18[0.93-1.52]$ & 0.175 & 1.15 [0.99-1.33] & 0.060 & & \\
\hline$\geq 40$ & $1.47[0.92-2.35]$ & 0.106 & $1.66[1.02-2.70]$ & 0.040 & $1.13[0.83-1.54]$ & 0.259 & & \\
\hline$<20$ & $1.53[1.17-2.01]$ & 0.002 & $1.77[1.27-2.46]$ & 0.001 & $1.28[1.07-1.53]$ & 0.006 & & \\
\hline \multicolumn{9}{|l|}{ Marital status } \\
\hline Currently married & 1.00 & & & & 1.00 & & & \\
\hline Formerly married $^{+}$ & $1.27[0.87-1.84]$ & 0.211 & & & 1.18 [0.94-1.48] & 0.149 & & \\
\hline Never married & $0.78[0.53-1.15]$ & 0.209 & & & $0.80[0.57-1.11]$ & 0.184 & & \\
\hline \multicolumn{9}{|l|}{ Birth order } \\
\hline First-born & 1.00 & & & & 1.00 & & & \\
\hline 2 nd -4 th & $0.96[0.74-1.24]$ & 0.736 & & & $1.04[0.89-1.22]$ & 0.602 & & \\
\hline $5+$ & $1.03[0.77-1.36]$ & 0.854 & & & $1.10[0.92-1.31]$ & 0.302 & & \\
\hline \multicolumn{9}{|l|}{ Preceding birth interval } \\
\hline No previous birth & 1.00 & & & & 1.00 & & & \\
\hline$<24$ months & $1.09[0.75-1.59]$ & 0.644 & & & $1.25[0.99-1.58]$ & 0.066 & & \\
\hline$>24$ months & $0.97[0.75-1.24]$ & 0.792 & & & $1.04[0.89-1.20]$ & 0.651 & & \\
\hline \multicolumn{9}{|l|}{ Place of delivery } \\
\hline Home & 1.00 & & & & 1.00 & & & \\
\hline Health facility & $0.81[0.65-1.00]$ & 0.053 & & & $0.76[0.65-0.88]$ & $<.0001$ & & \\
\hline
\end{tabular}


Table 2 Factors associated with stunting in children aged 0-23 months and 0-59 months (Continued)

\begin{tabular}{|c|c|c|c|c|c|c|c|c|}
\hline \multicolumn{9}{|l|}{ Type of delivery assistance } \\
\hline Health professional & 1.00 & & & & 1.00 & & & \\
\hline Traditional birth attendant & $1.44[1.09-1.91]$ & 0.010 & & & $1.55[1.28-1.88]$ & $<0.001$ & & \\
\hline Relatives or other & $1.28[0.99-1.64]$ & 0.052 & & & 1.33 [1.13-1.57] & 0.001 & & \\
\hline No one & $0.61[0.32-1.16]$ & 0.130 & & & $0.96[0.68-1.37]$ & 0.831 & & \\
\hline \multicolumn{9}{|l|}{ Mode of delivery } \\
\hline Non-caesarean & 1.00 & & & & 1.00 & & & \\
\hline Caesarean & $0.75[0.48-1.15]$ & 0.188 & & & $0.70[0.51-0.98]$ & 0.035 & & \\
\hline \multicolumn{9}{|l|}{ Timing of postnatal check-up } \\
\hline No check-ups ${ }^{\&}$ & 1.00 & & & & 1.00 & & & \\
\hline $0-2$ days & $0.95[0.73-1.25]$ & 0.729 & & & $0.85[0.70-1.03]$ & 0.101 & & \\
\hline 3-6 days & $1.04[0.67-1.59]$ & 0.874 & & & $0.99[0.72-1.35]$ & 0.927 & & \\
\hline $7+$ days & $0.71[0.51-0.98]$ & 0.040 & & & $0.73[0.57-0.92]$ & 0.011 & & \\
\hline \multicolumn{9}{|l|}{ Antenatal clinic visits } \\
\hline None & 1.00 & & & & 1.00 & & 1.00 & \\
\hline $1-3$ & $0.62[0.39-0.98]$ & 0.043 & & & $0.75[0.65-0.87]$ & $<0.001$ & $0.78[0.65-0.95]$ & 0.017 \\
\hline $4+$ & $0.59[0.38-0.92]$ & 0.020 & & & $0.71[0.61-0.84]$ & $<0.001$ & $0.82[0.65-1.02]$ & 0.080 \\
\hline \multicolumn{9}{|l|}{ Maternal BMI $\left(\mathrm{kgm}^{-2}\right)$} \\
\hline$>18.5$ & 1.00 & & & & 1.00 & & 1.00 & \\
\hline$<18.5$ & 1.54 [1.17-2.03] & 0.002 & & & $1.46[1.21-1.77]$ & $<0.001$ & $1.38[1.12-1.69]$ & 0.002 \\
\hline \multicolumn{9}{|l|}{ Child BF status } \\
\hline Exclusive BF & 1.00 & & & & 1.00 & & 1.00 & \\
\hline $\mathrm{BF}+$ water & $0.94[0.59-1.70]$ & 0.836 & & & $1.04[0.68-1.59]$ & 0.869 & $1.09[0.71-1.67]$ & 0.668 \\
\hline BF + supplements & $2.11[1.51,2.94]$ & $<0.001$ & & & $1.20[0.98-1.46]$ & 0.076 & $1.26[1.03-1.53]$ & 0.022 \\
\hline No BF & $5.07[3.40-7.56]$ & $<0.001$ & & & 1.69 [1.38-2.06] & $<0.001$ & $2.02[1.65-2.46$ & $<0.001$ \\
\hline \multicolumn{9}{|l|}{ Mother is literate } \\
\hline No & 1.00 & & 1.00 & & 1.00 & & & \\
\hline Yes & $0.93[0.77-1.12]$ & 0.450 & $1.36[1.03-1.82]$ & 0.032 & $0.82[0.72-0.93]$ & 0.003 & & \\
\hline \multicolumn{9}{|l|}{ Mother read newspaper } \\
\hline No & 1.00 & & & & 1.00 & & & \\
\hline Yes & $0.93[0.70-1.24]$ & 0.625 & & & $0.87[0.71-1.07]$ & 0.183 & & \\
\hline \multicolumn{9}{|l|}{ Mother listened to the radio } \\
\hline No & 1.00 & & & & 1.00 & & & \\
\hline Yes & 1.06 [0.87-1.29] & 0.537 & & & $\begin{array}{l}0.92 \\
{[0.81-1.04]}\end{array}$ & 0.190 & & \\
\hline \multicolumn{9}{|l|}{ Mother watched television } \\
\hline No & 1.00 & & & & 1.00 & & & \\
\hline Yes & $0.79[0.57-1.08]$ & 0.141 & & & $0.63[0.50-0.81]$ & $<0.001$ & & \\
\hline \multicolumn{9}{|l|}{ Child level factors } \\
\hline Child's age & 1.11 [1.09-1.13] & $<0.001$ & $1.11[1.10-1.13]$ & & $1.01[1.01-1.02]$ & $<0.001$ & & \\
\hline \multicolumn{9}{|l|}{ Sex of baby } \\
\hline Female & 1.00 & & 1.00 & & 1.00 & & 1.00 & \\
\hline Male & 1.42 [1.17-1.73] & $<0.001$ & 1.66 [1.34-2.06] & $<0.001$ & $1.36[1.21-1.52]$ & $<0.001$ & 1.39 [1.23-1.58] & $<0.001$ \\
\hline
\end{tabular}


Table 2 Factors associated with stunting in children aged 0-23 months and 0-59 months (Continued)

\begin{tabular}{|c|c|c|c|c|c|c|c|c|}
\hline \multicolumn{9}{|l|}{ Size of baby } \\
\hline Large & 1.00 & & 1.00 & & 1.00 & & 1.00 & \\
\hline Average & $1.30[1.03-1.64]$ & 0.028 & $1.39[1.09-1.77]$ & 0.007 & $1.23[1.07-1.40]$ & 0.002 & $1.18[1.03-1.34]$ & 0.015 \\
\hline Very small & $1.93[1.33-2.81]$ & $<0.001$ & $2.23[1.47-3.38]$ & $<0.001$ & $1.82[1.45-2.29]$ & $<0.001$ & $1.92[1.52-2.43]$ & $<0.001$ \\
\hline \multicolumn{9}{|c|}{ Child had diarrhoea (past 2 weeks) } \\
\hline No & 1.00 & & & & 1.00 & & & \\
\hline Yes & $1.11[0.87-1.41]$ & 0.407 & & & $1.03[0.87-1.22]$ & 0.715 & & \\
\hline \multicolumn{9}{|c|}{ Child had fever in (past two weeks) } \\
\hline No & 1.00 & & & & 1.00 & & & \\
\hline Yes & $1.19[0.96-1.46]$ & 0.115 & & & $1.02[0.88-1.18]$ & 0.754 & & \\
\hline \multicolumn{9}{|l|}{ Household level factors } \\
\hline \multicolumn{9}{|l|}{ Household wealth index } \\
\hline Richest & 1.00 & & & & 1.00 & & 1.00 & \\
\hline Richer & $1.35[0.89-2.04]$ & 0.155 & & & $1.68[1.25-2.25]$ & 0.001 & $1.40[1.03-1.89]$ & 0.030 \\
\hline Middle & $1.49[0.99-2.26]$ & 0.057 & & & $2.06[1.55-2.74]$ & $<0.001$ & $1.67[1.23-2.28]$ & 0.001 \\
\hline Poorer & $1.49[0.99-2.25]$ & 0.057 & & & $2.12[1.58-2.83]$ & $<0.001$ & $1.81[1.34-2.45]$ & $<0.001$ \\
\hline Poorest & $1.85[1.25-2.74]$ & 0.002 & & & $2.48[1.87-3.29]$ & $<0.001$ & $1.95[1.43-2.65]$ & $<0.001$ \\
\hline \multicolumn{9}{|l|}{ Source of drinking water } \\
\hline Protected & 1.00 & & 1.00 & & 1.00 & & 1.00 & \\
\hline Unprotected & $1.48[1.19-1.84]$ & $<0.001$ & $1.33[1.04-1.70]$ & 0.020 & $1.42[1.23-1.63]$ & $<0.001$ & $1.26[1.08-1.46]$ & 0.002 \\
\hline \multicolumn{9}{|l|}{ Community level factors } \\
\hline \multicolumn{9}{|l|}{ Type of residence } \\
\hline Urban & 1.00 & & & & 1.00 & & & \\
\hline Rural & $1.40[1.06-1.85]$ & 0.019 & & & $1.76[1.43-2.17]$ & $<0.001$ & & \\
\hline \multicolumn{9}{|l|}{ Geographic Zones } \\
\hline Northern & 1.00 & & & & 1.00 & & & \\
\hline Eastern & $0.84[0.51-1.37]$ & 0.486 & & & $0.62[0.44-0.86]$ & 0.005 & & \\
\hline Western & $1.35[0.96-1.90]$ & 0.085 & & & $0.95[0.75-1.19]$ & 0.640 & & \\
\hline Southern Highlands & $1.59[1.05-2.39]$ & 0.026 & & & 1.39 [1.01-1.90] & 0.043 & & \\
\hline Lake & $0.93[0.67-1.28]$ & 0.654 & & & $0.83[0.65-1.05]$ & 0.124 & & \\
\hline Southern & $1.15[0.78-1.71]$ & 0.482 & & & $1.08[0.85-1.38]$ & 0.527 & & \\
\hline Central & 1.55 [1.09-2.19] & 0.014 & & & 1.30 [0.99-1.70] & 0.063 & & \\
\hline Zanzibar & $0.81[0.58-2.18]$ & 0.222 & & & $0.55[0.43-0.71]$ & $<0.001$ & & \\
\hline
\end{tabular}

\&(including missing)

${ }^{+}$(divorced/separated /widowed)

although a comprehensive set of variables were used in our analysis, residual confounding from unmeasured covariates could not be ruled out.

Our study found that children in the 0-23 month age bracket had a significantly lower risk of being stunted compared to those in the older age bracket (0-59 months). Similar findings were reported by a recent study [18]. This finding may be due to the protective effect of breastfeeding, since almost all children in Tanzania are breastfed and most of them continue to be breastfed throughout the first year of their life [19]. The high risk of stunting observed beyond the 0-23 months-period may be linked to inappropriate food supplementation during the weaning period [20].

Children whose parents had no schooling were found to have a relatively higher risk of being stunted or severely stunted. This finding is consistent with those found in previous studies [20-23] in which stunting and severe stunting were positively associated with lower levels of parental education, which may be explained by the resulting limited family income and the consequent inadequate individual care and attention given to the child. Educated mothers would be more conscious about their children's health. 
Table 3 Factors associated with severe stunting in children aged 0-23 months and 0-59 months

\begin{tabular}{|c|c|c|c|c|c|c|c|c|}
\hline \multirow[t]{2}{*}{ Characteristic } & \multicolumn{4}{|c|}{ Severely stunted children 0-23 Months } & \multicolumn{4}{|c|}{ Severely stunted children 0-59 Months } \\
\hline & $\begin{array}{l}\text { Unadjusted OR } \\
{[95 \% \mathrm{Cl}]}\end{array}$ & $P$ & $\begin{array}{l}\text { Adjusted OR } \\
{[95 \% \text { Cl] }}\end{array}$ & $p$ & $\begin{array}{l}\text { Unadjusted OR } \\
{[95 \% \mathrm{Cl}]}\end{array}$ & $p$ & $\begin{array}{l}\text { Adjusted OR } \\
{[95 \% \mathrm{Cl}]}\end{array}$ & $p$ \\
\hline \multicolumn{9}{|l|}{ Parental factor } \\
\hline \multicolumn{9}{|l|}{ Maternal working status } \\
\hline Non-working & 1.00 & & & & 1.00 & & & \\
\hline $\begin{array}{l}\text { Working } \\
\text { (past } 12 \text { months) }\end{array}$ & $1.15[0.77-1.70]$ & 0.497 & & & $1.05[0.78-1.40]$ & 0.761 & & \\
\hline \multicolumn{9}{|l|}{ Maternal education } \\
\hline Secondary and above & 1.00 & & 1.00 & & 1.00 & & 1.00 & \\
\hline Primary & $3.44[1.68-7.02]$ & 0.001 & $3.63[1.58-8.28]$ & 0.002 & $3.28[2.04-5.27]$ & $<0.001$ & $1.95[1.12-3.41]$ & 0.017 \\
\hline No education & $4.41[2.22-8.76]$ & $<0.001$ & $4.86[2.08-11.35]$ & $<0.001$ & $4.50[2.74-7.39]$ & $<0.001$ & $2.57[1.46-4.50]$ & 0.001 \\
\hline \multicolumn{9}{|l|}{ Partner's occupation } \\
\hline Non agriculture & 1.00 & & & & 1.00 & & & \\
\hline Agriculture & $1.43[1.01-2.03]$ & 0.046 & & & $1.50[1.22-1.84]$ & $<0.001$ & & \\
\hline Not working & $0.73[0.38-1.41]$ & 0.352 & & & $1.08[0.72-1.61]$ & 0.721 & & \\
\hline \multicolumn{9}{|l|}{ Partner's education } \\
\hline Secondary and above & 1.00 & & & & 1.00 & & 1.00 & \\
\hline Primary & $1.99[1.16-3.44]$ & 0.013 & & & $2.57[1.80-3.66]$ & $<0.001$ & 1.79 [1.19-2.71] & 0.005 \\
\hline No education & $1.87[1.03-3.40]$ & 0.041 & & & 2.92 [1.94-4.39] & $<0.001$ & $1.71[1.07-2.72]$ & 0.022 \\
\hline \multicolumn{9}{|l|}{ Mother's age } \\
\hline $15-24$ years & 1.00 & & & & 1.00 & & & \\
\hline $25-34$ years & $0.98[0.73-1.30]$ & 0.873 & & & $1.14[0.92-1.42]$ & 0.212 & & \\
\hline $35-49$ years & $1.46[0.99-2.14]$ & 0.054 & & & $1.27[0.96-1.66]$ & 0.089 & & \\
\hline \multicolumn{9}{|l|}{ Mother's age at birth } \\
\hline$<19$ years & 1.00 & & & & 1.00 & & & \\
\hline 20-29 years & $0.61[0.43-0.88]$ & 0.009 & & & $0.87[0.67-1.12]$ & 0.293 & & \\
\hline 30-39 years & $0.97[0.64-1.47]$ & 0.893 & & & $1.08[0.81-1.45]$ & 0.597 & & \\
\hline 40 and above & $0.88[0.44-1.76]$ & 0.726 & & & $0.93[0.57-1.52]$ & 0.774 & & \\
\hline \multicolumn{9}{|l|}{ Marital status } \\
\hline Currently married & 1.00 & & & & 1.00 & & & \\
\hline Formerly married $^{+}$ & $1.49[0.93-2.39]$ & 0.099 & & & $1.33[0.98-1.79]$ & 0.064 & & \\
\hline Never married & $0.60[0.33-1.10]$ & 0.101 & & & $0.80[0.52-1.22]$ & 0.301 & & \\
\hline \multicolumn{9}{|l|}{ Birth order } \\
\hline First-born & 1.00 & & & & 1.00 & & & \\
\hline 2nd -4th & $0.94[0.65-1.35]$ & 0.734 & & & $1.24[0.98-1.56]$ & 0.074 & & \\
\hline 5 or more & $1.06[0.71-1.57]$ & 0.781 & & & $1.34[1.02-1.72]$ & 0.034 & & \\
\hline \multicolumn{9}{|l|}{ Preceding birth interval } \\
\hline No previous birth & 1.00 & & & & 1.00 & & & \\
\hline$<24$ months & $1.28[0.80-2.04]$ & 0.296 & & & $1.52[1.15-2.02]$ & 0.003 & & \\
\hline$>24$ months & $0.94[0.65-1.34]$ & 0.721 & & & $1.23[0.97-1.54]$ & 0.081 & & \\
\hline \multicolumn{9}{|l|}{ Place of delivery } \\
\hline Home & 1.00 & & & & 1.00 & & & \\
\hline Health facility & $0.68[0.52-0.91]$ & 0.010 & & & $0.68[0.57-0.83]$ & $<0.001$ & & \\
\hline
\end{tabular}


Table 3 Factors associated with severe stunting in children aged 0-23 months and 0-59 months (Continued)

Mode of delivery

Non-caesarean

Caesarean

1.00

$0.77[0.34-1.72] \quad 0.520$

Type of delivery assistance

$\begin{array}{lll}\text { Health professional } & 1.00 & \\ \text { Traditional birth attendant } & 1.70[1.19-2.42] & 0.003 \\ \text { Relatives and other } & 1.57[1.14-2.19] & 0.006 \\ \text { No one } & 0.89[0.34-2.34] & 0.816\end{array}$

Antenatal clinic visits

$$
\begin{aligned}
& 4+ \\
& 1-3 .
\end{aligned}
$$

None

Timing of postnatal check-up

$\begin{array}{lll}\text { No check-ups }{ }^{\&} & 1.00 & \\ 0-2 \text { days } & 0.94[0.66-1.34] & 0.726 \\ 3-6 \text { days } & 1.11[0.64-1.95] & 0.703 \\ 7+\text { days } & 0.75[0.48-1.17] & 0.207\end{array}$

Maternal BMI

$$
\begin{aligned}
& >18.5\left(\mathrm{~kg} / \mathrm{m}^{2}\right) \\
& <=18.5\left(\mathrm{~kg} / \mathrm{m}^{2}\right)
\end{aligned}
$$

1.00

$1.77[1.20-2.62]$

0.004

1.00

$1.59[1.05-2.41]$

1.00

0.84 [0.29-2.45]

0.756

$1.74[1.04-2.92]$

0.035

BF + supplements

3.07 [1.80-5.23]

$<0.001$

Mother was literate

$$
\begin{array}{lll}
\text { No } & 1.00 \\
\text { Yes } & 0.85[0.65-1.09] & 0.209
\end{array}
$$

Listening to radio

$\begin{array}{lll}\text { No } & 1.00 \\ \text { Yes } & 0.89[0.67-1.18] & 0.445\end{array}$

Mother read newspaper/magazine

$\begin{array}{lll}\text { No } & 1.00 & \\ \text { Yes } & 0.81[0.55-1.19] & 0.292\end{array}$

Mother watched TV

$\begin{array}{lll}\text { No } & 1.00 & \\ \text { Yes } & 0.79[0.51-1.21] & 0.278\end{array}$

Child level factors

Child's age

$1.06[1.09-1.11]<0.001$

$$
1.09 \text { [1.07-1.12] }
$$$$
<0.001
$$

0.199

1.00

$1.25[1.01-1.54] \quad 0.043$

$1.38[1.11-1.72] \quad 0.004$

1.00

1.88 [1.46-2.43]

1.57 [1.27-1.94]

0.79 [0.47-1.33]

1.00

$<0.001$

0.385

1.51 [1.15-1.99] 0.003

$1.34[1.06-1.70] \quad 0.014$

$0.78[0.44-1.39] \quad 0.409$

Sex of baby

Female

Male
1.00

$1.46[1.13-1.89] \quad 0.003$
1.00

1.63 [1.22-2.16]
1.00

0.82 [0.64-1.06] $\quad 0.124$

$1.11[0.70-1.72] \quad 0.684$

0.84 [0.63-1.12] $\quad 0.239$

1.00

$1.67[1.28-2.19]$

$<0.001$

1.00

1.00

$0.71[0.34-1.36] \quad 0.303$

$0.96[0.76-1.27] \quad 0.772$

$1.16[0.89-1.51] \quad 0.270$

1.00

$0.71[0.60-0.85]<0.001$

1.00

$0.80[0.67-0.94] \quad 0.009$

1.00

$0.82[0.64-1.04] \quad 0.114$

1.00

$0.67[0.50-0.90] \quad 0.007$

$1.00[1.00-1.01] \quad 0.002$

1.00

1.00

$1.36[1.17-1.58] \quad<0.001 \quad 1.45[1.23-1.72] \quad<0.001$ 
Table 3 Factors associated with severe stunting in children aged 0-23 months and 0-59 months (Continued)

\begin{tabular}{|c|c|c|c|c|c|c|c|c|}
\hline \multicolumn{9}{|l|}{ Size of baby } \\
\hline Large & 1.00 & & 1.00 & & 1.00 & & 1.00 & \\
\hline Average & $1.60[1.15-2.24]$ & 0.005 & $1.65[1.18-2.31]$ & 0.004 & $1.55[1.25-1.90]$ & $<0.001$ & $1.48[1.19-1.84]$ & $<0.001$ \\
\hline Very small & $3.06[1.77-5.29]$ & $<0.001$ & $3.26[1.80-5.90]$ & $<0.001$ & $2.54[1.88-3.43]$ & $<0.001$ & $2.64[1.92-3.63]$ & $<0.001$ \\
\hline \multicolumn{9}{|c|}{ Child had diarrhoea in the last 2 weeks } \\
\hline No & 1.00 & & & & 1.00 & & & \\
\hline Yes & $1.06[0.75-1.51]$ & 0.738 & & & $1.15[0.92-1.42]$ & 0.212 & & \\
\hline \multicolumn{9}{|c|}{ Child had fever in last 2 weeks } \\
\hline No & 1.00 & & & & 1.00 & & & \\
\hline Yes & $1.19[0.87-1.62]$ & 0.272 & & & $0.99[0.78-1.25]$ & 0.941 & & \\
\hline \multicolumn{9}{|l|}{ Household level factors } \\
\hline \multicolumn{9}{|l|}{ Wealth Index } \\
\hline Poorest & 1.00 & & & & 1.00 & & & \\
\hline Poorer & $1.01[0.56-1.82]$ & 0.979 & & & $1.50[0.96-2.35]$ & 0.074 & & \\
\hline Middle & $1.18[0.64-2.18]$ & 0.585 & & & $1.77[1.19-2.63]$ & 0.005 & & \\
\hline Rich & $1.51[0.86-2.65]$ & 0.147 & & & $2.29[1.54-3.41]$ & $<0.001$ & & \\
\hline Richest & $1.52[0.85-2.75]$ & 0.159 & & & $2.39[1.59-3.58]$ & $<0.001$ & & \\
\hline \multicolumn{9}{|l|}{ Source of drinking water } \\
\hline Protected & 1.00 & & 1.00 & & 1.00 & & 1.00 & \\
\hline Unprotected & $1.58[1.15-2.18]$ & 0.005 & $1.50[1.05-2.14]$ & 0.025 & $1.58[1.29-1.94]$ & $<0.001$ & $1.22[1.13-1.79]$ & 0.003 \\
\hline \multicolumn{9}{|l|}{ Community level factors } \\
\hline \multicolumn{9}{|l|}{ Type of residence } \\
\hline Rural & 1.00 & & 1.00 & & 1.00 & & & \\
\hline Urban & $1.06[0.74-1.53]$ & 0.734 & $1.52[1.02-2.27]$ & 0.040 & $1.54[1.16-2.05]$ & 0.003 & & \\
\hline \multicolumn{9}{|l|}{ Geographic Zones } \\
\hline Northern & 1.00 & & & & 1.00 & & 1.00 & \\
\hline Eastern & $0.81[0.40-1.60]$ & 0.530 & & & $0.65[0.35-1.03]$ & 0.065 & $0.74[0.43-1.27]$ & 0.281 \\
\hline Western & $1.09[0.69-1.73]$ & 0.692 & & & $0.70[0.48-1.02]$ & 0.065 & $0.64[0.42-0.95]$ & 0.028 \\
\hline Southern Highlands & $1.28[0.76-2.15]$ & 0.351 & & & $1.15[0.77-1.71]$ & 0.473 & $1.13[0.75-1.71]$ & 0.546 \\
\hline Lake & $0.78[0.45-1.37]$ & 0.389 & & & $0.74[0.48-1.16]$ & 0.199 & $0.73[0.46-1.13]$ & 0.164 \\
\hline Southern & $0.98[0.57-1.70]$ & 0.961 & & & $0.97[0.66-1.43]$ & 0.888 & $0.96[0.65-1.43]$ & 0.876 \\
\hline Central & $1.53[0.91-2.56]$ & 0.105 & & & 1.31 [0.89-1.92] & 0.165 & $1.15[0.77-1.70]$ & 0.484 \\
\hline Zanzibar & $0.93[0.57-1.50]$ & 0.765 & & & $0.59[0.40-0.86]$ & 0.007 & $0.80[0.52-1.24]$ & 0.327 \\
\hline
\end{tabular}

\&(including missing)

${ }^{+}$(divorced/separated /widowed)

Children whose mothers perceived them to be small or very small at birth were found to be at a relatively higher risk of being stunted compared to other children in this age group. A similar association between birth weight, which has been found to be a measure of perceived size of the baby [24] and later risk for stunting has previously been documented in other low-income countries [15, 25]. Such children were found to be associated with severe stunting, consistent with previous other studies [25-27]. Small newborns from less affluent areas thus do not seem to demonstrate marked catch-up growth during infancy. As the prevalence of low birth weight $(<2500 \mathrm{~g})$ is as high as $16 \%$ in Tanzania [19], prevention of intrauterine growth retardation and preterm births must form one cornerstone in the population level management strategy for infant stunting. Assessment of the size of the baby at birth may be important for health care providers since this can be used to identify the risk of stunting among children in order to take necessary measures.

In this current study, male children aged 0-59 months were found to have a higher risk of being stunted or severely stunted, compared to females. This finding is 
consistent with a finding reported from a meta-analysis of sixteen demographic and health surveys of ten countries in sub-Saharan Africa, in which male children were found to be consistently more likely to become stunted compared to their female counterparts [28]. A recent study also associated male children with severe stunting [26]. These sex differences in stunting and severe stunting could be explained by behavioural patterns employed by communities such as favouritism which may involve dietary intakes towards daughters. In a previous study, it was reported that males were given supplemental foods earlier, were fed larger quantities of supplemental foods and had higher rates of diarrhoea compared to females [29].

Our study found that children whose mothers had no schooling were more likely to become stunted compared to those whose mothers had secondary education or higher. This reflects the importance of education for mothers in regard to the development of healthy children, as reported by previous studies [10].

In the present study, children from poorest households were found to have a significantly higher risk of being stunted compared to those from the middle-income, rich and richest households. The effect of wealth on stunting can be explained by its importance in the purchase of food and consumer goods that promote and protect the health of children. Various studies have observed a positive association between low income and malnutrition $[10,30,31]$, which often leads to stunting.

In our analyses, children born to mothers of low BMI were more likely to be severely stunted compared to those born to mothers with higher BMI. Previous studies have associated stunting with maternal factors and in particular the mother's poor nutritional status before conception and poor nutrition during pregnancy [15, 32, 33]. Sufficient weight gain during pregnancy is particularly important since it accounts for a large proportion of foetal growth retardation.

The risk of stunting was found to be significantly higher among children who were no longer breastfeeding and those who were breastfed longer than 12 months. A recent study in Ecuador [34] revealed that children who were stunted made up $30 \%$ of those exclusively breastfed for less than or equal to 6 months, $23.3 \%$ of the children exclusively breastfed for between 6-12 months and $27.7 \%$ of those exclusively breastfed for 12 months or longer. Although the WHO recommends that women exclusively breastfeed their children for 6 months, there has been evidence that breastfeeding alone may not adequately meet the nutritional requirements of a 6 month-old baby [35]. If this is indeed the case, it may be likely that exclusively breastfeeding for 12 months or longer does not provide enough energy for growing babies in Tanzania. Our study also found that infants who were not breastfed were significantly associated with stunting and severe stunting among children from both age groups. This finding is consistent with previous studies [36-38].

In our study, we found that children born to relatively younger mothers ( $<20$ years) had a significantly higher risk of being stunted. This may be attributed to the fact that such mothers would not have the requisite experience or knowledge to provide the child with the proper care. However, a previous study reported that mother's age at pregnancy is not a predictor of stunting [39].

Being born at home and delivered by TBAs were found to be significant risk factors to severe stunting for children aged 0-59 months. This finding is consistent with a previous study in rural Malawi [26]. Mothers of such children may not have had any contacts with trained medical professionals to receive proper advice on appropriate child feeding practices.

\section{Conclusions}

This current study has highlighted the individual-, household- and community-level factors associated with stunting and severe stunting among Tanzanian children. The main risk factors included male children, children perceived to be small at birth, children from poorest households with no potable water and those who were born at home with assistance from traditional birth attendants. Our findings indicate the need for interventions at both the individual and community levels. Peer-based community interventions including peer-education, where older and more experienced women could educate these young mothers about appropriate child feeding practices aimed at long-term prevention of stunting and severe stunting in Tanzania are required to improve child health. At the individual level, emphasis should be placed on educating mothers and particularly young mothers regarding health and child feeding practices including safe sources of drinking water for their children.

\section{Abbreviations}

WHO: World Health Organization; TDHS: Tanzania Demographic and Health Survey; SD: standard deviation; TBAs: Traditional Birth Attendants; BMI: Body Mass index; OR: Odds ratio; Cl: Confidence interval.

\section{Competing interests}

The authors declare that they have no competing interests.

\section{Authors' contributions}

LC and KEA designed the study, performed the analysis and prepared the manuscript. DC, HM, RV, SK, IAI, SKB and M.JD provided revision of the final manuscript. All authors read and approved the manuscript.

\section{Acknowledgements}

The authors gratefully acknowledge the Australian Leadership Award Fellowships Programme of the Australian Agency for International Development (AusAID) for financing 11 researchers from Tanzania. 


\section{Author details}

${ }^{1}$ Muhimbili University of Health and Allied Sciences, Dar es Salaam, Tanzania. ${ }^{2}$ Tanzania Food and Nutrition Centre, Dar es Salaam, Tanzania. ${ }^{3}$ Sokoine University of Agriculture, Morogoro, Tanzania. ${ }^{4}$ School of Health Sciences, University of Newcastle, New South Wales, Australia. ${ }^{5}$ Sydney School of Public Health, University of Sydney, New South Wales, Australia. ${ }^{6}$ School of Science and Health, Western Sydney University, Building 24.2.40, Campbelltown Campus, Locked Bag 1797, Penrith, NSW 2571, Australia.

\section{Received: 1 April 2015 Accepted: 8 October 2015}

\section{Published online: 21 October 2015}

\section{References}

1. UNICEF. Division of Communication. Tracking progress on child and maternal nutrition: a survival and development priority. UNICEF, 2009. http://www.unicef.org/publications/index_51656.html (accessed March, 2015).

2. Scrimshaw NS, SanGiovanni JP. Synergism of nutrition, infection, and immunity: an overview. Am J Clin Nutr. 1997;66:464S-77.

3. Black RE, Allen LH, Bhutta ZA, Caulfield LE, Onis MD, Ezzati M. Maternal and child undernutrition: global and regional exposures and health consequences. Lancet. 2008:371:243-60.

4. Willey BA, Cameron N, Norris SA, Pettifor JM, Griffiths PL. Socio-economic predictors of stunting in preschool children: a population-based study from Johannesburg and Soweto. S Afr Med J. 2009;99:450-6.

5. Victora CG, de Onis M, Hallal PC, Blössner M, Shrimpton R. Worldwide timing of growth faltering: revisiting implications for interventions. Pediatrics. 2010:peds. 2009-1519. doi: 10.1542/peds.2009-1519. Epub 2010 Feb 15.

6. De Onis M, Blössner M, Borghi E. Prevalence and trends of stunting among pre-school children, 1990-2020. Public Health Nutr. 2012;15:142-8.

7. National Bureau of Statistics, ICF Macro. Tanzania Demographic and Health Survey 2010. Dar es Salaam, Tanzania 2011

8. Marjan ZM, Kandiah M, Lin KG, Siong TE. Socioeconomic profile and nutritional status of children in rubber smallholdings. Asia Pac J Clin Nutr. 2002;11:133-41.

9. Ukwuani FA, Suchindran CM. Implications of women's work for child nutritional status in sub-Saharan Africa: a case study of Nigeria. Soc Sci Med. 2003;56:2109-21.

10. Delpeuch F, Traissac P, Martin-Prével Y, Massamba J, Maire B. Economic crisis and malnutrition: socioeconomic determinants of anthropometric status of preschool children and their mothers in an African urban area. Public Health Nutr. 2000;3:39-47.

11. Saleemi M, Ashraf R, Mellander L, Zaman S. Determinants of stunting at 6, 12, 24 and 60 months and postnatal linear growth in Pakistani children. Acta Paediatr. 2001;90:1304-8.

12. Hernandez-Diaz S, Peterson K, Dixit S, Hernandez B, Parra S, Barquera S, et al. Association of maternal short stature with stunting in Mexican children: common genes vs common environment. Eur J Clin Nutr. 1999;53:938-45.

13. Teshome B, Kogi-Makau W, Getahun Z, Taye G. Magnitude and determinants of stunting in children underfive years of age in food surplus region of Ethiopia: the case of west gojam zone. Ethiop J Health Dev. 2009;23.

14. Espo M, Kulmala T, Maleta K, Cullinan T, Salin ML, \& Ashorn P. Determinants of linear growth and predictors of severe stunting during infancy in rural Malawi. Acta Paediatrica, 2002; 91(12), 1364-1370

15. Mamiro PS, Kolsteren P, Roberfroid D, Tatala S, Opsomer AS, Van Camp JH. Feeding practices and factors contributing to wasting, stunting, and iron-deficiency anaemia among 3-23-month old children in Kilosa district, rural Tanzania. J Health Popul Nutr. 2005;222-230.

16. Abubakar A, Uriyo J, Msuya SE, Swai M, Stray-Pedersen B. Prevalence and risk factors for poor nutritional status among children in the Kilimanjaro Region of Tanzania. Int J Environ Res Public Health. 2012;9:3506-18.

17. Filmer D, Pritchett LH. Estimating Wealth Effects Without Expenditure Data-Or Tears: An Application To Educational Enrollments In States Of India*. Demography. 2001;38:115-32.

18. Hien NN, Kam S. Nutritional status and the characteristics related to malnutrition in children under five years of age in Nghean, Vietnam. J Prev Med Public Health. 2008:41:232-40

19. Tanzania U. Tanzania Demographic and Health Survey 2010. DHS Dar es Salaam: National Bureau of Statistics, Dar es Salaam, Tanzania \& Macro; 2011.

20. Mittal A, Singh J, Ahluwalia S. Effect of maternal factors on nutritional status of 1-5-year-old children in urban slum population. Indian J Community Med. 2007;32:264
21. Abuya B, Onsomu E, Kimani J, Moore D. Influence of maternal education on child immunization and stunting in Kenya. Matern Child Health J. 2011;15:1389-99.

22. Semba RD, de Pee S, Sun K, Sari M, Akhter N, Bloem MW. Effect of parental formal education on risk of child stunting in Indonesia and Bangladesh: a cross-sectional study. Lancet. 2008;371:322-8.

23. Wamani H, Tylleskär T, Åstrøm AN, Tumwine JK, Peterson S. Mothers' education but not fathers' education, household assets or land ownership is the best predictor of child health inequalities in rural Uganda. Int J Equity Health. 2004;3:9

24. Channon AA. Can mothers judge the size of their newborn? Assessing the determinants of a mother's perception of a baby's size at birth. J Biosoc Sci. 2011:43:555-73.

25. Arifeen S, Black R, Caulfield L, Antelman G, Baqui A. Determinants of infant growth in the slums of Dhaka: size and maturity at birth, breastfeeding and morbidity. Eur J Clin Nutr. 2001;55:167-78.

26. Ramli, Agho KE, Inder KJ, Bowe SJ, Jacobs J, \& Dibley MJ. Prevalence and Risk Factors for Stunting and Severe Stunting among Under-fives in North Maluku Province of Indonesia. Biomed Central (BMC) Pediatrics, 2009;9: 64.

27. Cohen RJ, Brown KH, Canahuati J, Rivera LL, Dewey KG. Determinants of growth from birth to 12 months among breast-fed Honduran infants in relation to age of introduction of complementary foods. Pediatrics. 1995;96:504-10.

28. Wamani H, Åstrøm AN, Peterson S, Tumwine JK, Tylleskär T. Boys are more stunted than girls in sub-Saharan Africa: a meta-analysis of 16 demographic and health surveys. BMC Pediatr. 2007;7:17.

29. Marcoux A. Sex differentials in undernutrition: A look at survey evidence. Popul Dev Rev. 2002;28:275-84

30. Reyes H, Pérez-Cuevas R, Sandoval A, Castillo R, Santos Jl, Doubova SV, et al. The family as a determinant of stunting in children living in conditions of extreme poverty: a case-control study. BMC Public Health. 2004;4:57.

31. Monteiro CA, Benicio MHDA, Conde WL, Konno S, Lovadino AL, Barros AJ, et al. Narrowing socioeconomic inequality in child stunting: the Brazilian experience, 1974-2007. Bull World Health Organ. 2010;88:305-11.

32. Semba RD. Piot P. Nutrition and health in developing countries. Springer: Bloem MW; 2008.

33. Dekker LH, Mora-Plazas M, Marín C, Baylin A, Villamor E. Stunting associated with poor socioeconomic and maternal nutrition status and respiratory morbidity in Colombian schoolchildren. Food \& Nutrition Bulletin. 2010;31:242-50.

34. Faldetta KF, Pujalte GGA. The Relationship between Exclusive Breastfeeding Duration and Growth in San Pablo, Ecuador. J Global Health Perspectives. 2012;1:1-5.

35. The World Bank. Nutrition at a glance: Ecuador. In Reference Link 2011 AJ ed. 2009.

36. Kumar D, Goel N, Mittal PC, Misra P. Influence of infant-feeding practices on nutritional status of under-five children. Indian J Pediatrics. 2006;73:417-21.

37. Bloss E, Wainaina F, Bailey RC. Prevalence and predictors of underweight, stunting, and wasting among children aged 5 and under in western Kenya. J Trop Pediatr. 2004:50:260-70.

38. Dewey KG, Cohen RJ. Does birth spacing affect maternal or child nutritional status? A systematic literature review. Matern Child Nutr. 2007:3:151--+73.

39. Varela-Silva Ml, Azcorra H, Dickinson F, Bogin B, Frisancho A. Influence of maternal stature, pregnancy age, and infant birth weight on growth during childhood in Yucatan, Mexico: a test of the intergenerational effects hypothesis. Am J Hum Biol. 2009;21:657-63. 